Article

\title{
Soft Open Bases and a Novel Construction of Soft Topologies from Bases for Topologies
}

\author{
José Carlos R. Alcantud (D) \\ BORDA Research Unit and Multidisciplinary Institute of Enterprise (IME), University of Salamanca, \\ E37007 Salamanca, Spain; jcr@usal.es
}

Received: 14 April 2020; Accepted: 27 April 2020; Published: 29 April 2020

check for updates

\begin{abstract}
Soft topology studies a structure on the collection of all soft sets on a given set of alternatives (the relevant attributes being fixed). It is directly inspired by the axioms of a topological space. This paper contributes to the theoretical bases of soft topology in various ways. We extend a general construction of soft topologies from topologies on the set of alternatives in two different directions. An extensive discussion with criteria about what a soft counterpart of "topological separability" should satisfy is also given. The interactions of the properties that arise with separability, and of second-countability and its soft counterpart, are studied under the general mechanisms that generate soft topological spaces. The first non-trivial examples of soft second-countable soft topological spaces are produced as a consequence.
\end{abstract}

Keywords: soft open base; soft topology; topology; separability; second countability axiom

\section{Introduction}

Soft topology stands at the junction of soft set theory [1] and topology [2,3]. It is concerned with a structure on the set of all soft sets, and is inspired by the standard axioms of a topological space.

Soft sets were introduced to describe a universe of alternatives by a set of relevant attributes. Soft set theory has been a thriving field of research and interaction with other disciplines ever since its establishment in 1999. Specifically, the field of soft topology was independently set up by the works of Çağman, Karataş and Enginoglu [4] and Shabir and Naz [5]. Although many sudies followed their directions, the growth of the literature has been irregular and there is a scant collection of keystone articles and results. We acknowledge the foremost role of [6-11] among others.

This article has several objectives. Its driving force is the construction of soft topologies on the set of alternatives from elements pertaining to standard topology theory (either a collection of topologies, or bases of topologies). We also propose some axioms of 'soft separability' and study their behavior and the theoretical properties of soft second-countability.

Let us give a more precise list of the research goals of this paper.

First, we improve a known process that produces a soft topology from one topology by the recourse to a family of topologies (one for each attribute). Despite its generality, we will show that this procedure interacts nicely with interesting axioms.

Secondly, we perform a specific analysis of what 'soft separability' should mean, as an extension of the usual concept of topological separability. Three criteria for the suitability of the soft counterpart(s) of topological separability are proposed. Then, two related concepts are put forward. They rely on completely different approaches to the idea of 'soft separability', however, they are not independent. Their relationship with topological separability is analyzed through the (improved) process that passes topologies on to soft topologies. Soft second-countability, which is quite indisputable as an axiom in soft topology, is strictly stronger than these concepts, and we prove by example that it is indeed different from them. 
From a theoretical standpoint, we prove a characterization of soft second-countable topologies that are generated by several topologies from the aforementioned procedure. When the set of attributes is at most countable, soft second-countability is equivalent to the fact that all the topologies associated with the attributes are second-countable.

Last but not least, we introduce a practical new procedure that produces soft topologies and depends upon a family of bases for topologies (one for each attribute). It allows us to visualize soft topologies in a simpler manner because bases are simpler than topologies and the same is true in the field of soft topologies. Another advantage is that it is a very natural way for the analysis of soft second-countability. Actually, with the help of this construction we put forward several non-trivial classes of (countable and uncountable) soft topological spaces with the soft second-countability property.

The remainder of this paper is organized as follows. Section 2 briefly recalls some basic notions concerning soft sets and soft topologies. We also show how a family of topologies (one for each attribute) can be used to generate a soft topology. Section 3 discusses the elusive concept of 'soft separability'. Here we make some new proposals and discuss why they are nice candidates for being accepted as suitable extensions of the concept of separability in topology. Section 4 addresses our characterization of soft second-countability of soft topologies generated by families of topologies. Section 5 introduces a novel construction of soft topologies from bases for a crisp topology. Finally, the last section summarizes this study and suggests possible future works.

\section{Preliminaries}

Henceforth, $\mathcal{P}(X)$ denotes the set of parts of a set $X$, i.e., $\mathcal{P}(X)$ is the set formed by all the subsets of $X$. In this section, we briefly recall the rudiments of soft set theory and soft topology. To emphasize the distinction between topologies and soft topologies, we sometimes use the term "crisp topology" to refer to a standard topology on a set. For the basic notions in (crisp) topology that we use, the reader can refer to Munkres [2] and Willard [3].

In this paper, $X$ is a fixed nonempty set, often called a universe of discourse, and $E$ is a set of attributes. Unless otherwise stated, henceforth $X$ and $E$ are unrestricted sets, i.e., they are possibly very large. We underline this feature because in almost all examples in the literature about soft topologies, both $X$ and $E$ are finite.

\subsection{Soft Sets and Their Operations}

According to [1], a soft set on $X$ is a pair $(F, E)$ where $F$ is a mapping $F: E \longrightarrow \mathcal{P}(X)$. It is assumed that set $E$ consists of all relevant attributes. Thus, a soft set over $X$ is regarded as a parameterized family of subsets of $X$. The collection of all soft sets on $X$ with attributes $E$ is denoted as $S S_{E}(X)$, or simply $S S(X)$ when $E$ is common knowledge.

When $e \in E$, the subset $F(e) \subseteq X$ is called the set of $e$-approximate elements of $X$, or alternatively, the subset of $X$ approximated by $e$.

Examples include the null $\Phi$ and the absolute $\tilde{X}$ soft sets on $X$. They satisfy $\Phi(e)=\varnothing$ for each $e \in E$, and $\tilde{X}(e)=X$ for each $e \in E$, respectively.

Soft sets on $X$ with $F(e)$ finite, resp., countable, for each $e \in E$ are called finite, resp., countable, soft sets on $X[8,9]$.

Soft points are also special soft sets. Two concepts have appeared in the literature under the same term $[8,11]$, but we only need one of them. Thus, in this paper, soft points are soft sets $(F, E)$ for which there exists $x \in X$ such that $F(e)=\{x\}$ for all $e \in E$, and we denote by $(\{x\}, E)$ this soft point [11]. Note that in [8] and elsewhere, the term soft points has also been used for soft sets $(F, E)$ for which there exists $x \in X$ and $e \in E$ such that $F(e)=\{x\}$ and $F\left(e^{\prime}\right)=\varnothing$ for all $e^{\prime} \in E \backslash\{e\}$.

Two notations make it easier to operate with soft sets.

(1) The soft set $(F, E)$ described above is usually represented as $\{(e, F(e)): e \in E\}$. 
(2) Assume a finite environment such that $X=\left\{x_{1}, x_{2}, \ldots, x_{m}\right\}$ and $E=\left\{e_{1}, e_{2}, \ldots, e_{n}\right\}$. Then the soft set $(F, E)$ can be represented in tabular form as in Figure 1 .

In this representation, the binary digit $r_{j k}$ is 1 when $x_{j} \in F\left(e_{k}\right)$ and it is 0 otherwise.

\begin{tabular}{ccccc}
$(F, E)$ & $e_{1}$ & $e_{2}$ & $\cdots$ & $e_{n}$ \\
\hline$x_{1}$ & $r_{11}$ & $r_{12}$ & $\cdots$ & $r_{1 n}$ \\
$x_{2}$ & $r_{21}$ & $r_{22}$ & $\cdots$ & $r_{2 n}$ \\
$\vdots$ & $\vdots$ & $\vdots$ & & $\vdots$ \\
$x_{m}$ & $r_{m 1}$ & $r_{m 2}$ & $\cdots$ & $r_{m n}$
\end{tabular}

Figure 1. The tabular representation of a soft set $(F, E)$.

Unions, intersections, inclusions and complements are standard set-theoretic operations on soft sets [12]. Unions and intersections of two soft sets on a common set $X$ are defined as follows (and their extensions to arbitrary collections of soft sets are immediate): when $\left(F_{1}, E\right),\left(F_{2}, E\right)$,

$\left(F_{1}, E\right) \sqcup\left(F_{2}, E\right)$ is $\left(F_{3}, E\right) \in S S_{E}(X)$ such that $F_{3}(e)=F_{1}(e) \cup F_{2}(e)$ for each $e \in E$, and $\left(F_{1}, E\right) \sqcap$ $\left(F_{2}, E\right)$ is $\left(F_{4}, E\right) \in S S_{E}(X)$ such that $F_{4}(e)=F_{1}(e) \cap F_{2}(e)$ for each $e \in E$.

We write $\left(F_{1}, E\right) \sqsubseteq\left(F_{2}, E\right)$ when $F_{1}(e) \subseteq F_{2}(e)$ for each $e \in E$.

Finally, the complement of $(F, E) \in S S_{E}(X)$ is $(F, E)^{c}=\left(F^{c}, E\right) \in S S_{E}(X)$ with the property that $F^{c}(e)=X \backslash F(e)$ for each $e \in E$. In the tabular representation, the complement swaps 0 's and 1 's elsewhere.

Aygünoğlu and Aygün [7], Theorem 2.1 summarize results on the behavior of these operators from $[12,13]$. They endow $S S_{E}(X)$ with the structure of a Boolean lattice.

\subsection{Soft Topologies, Soft Bases and Soft Open Bases}

The main concept in this section was independently proposed by Çağman, Karataş and Enginoglu [4] and Shabir and Naz [5].

Definition $1([4,5])$. A soft topology $\tau$ on $X$ is defined as a collection of soft sets on $X, \tau \in \mathcal{P}\left(S_{E}(X)\right)$, with the following properties:

(1) $\Phi, \tilde{X} \in \tau$;

(2) the union of soft sets in $\tau$ belongs to $\tau$; and

(3) the intersection of a finite number of soft sets in $\tau$ belongs to $\tau$.

In this definition, union means $\sqcup$ and intersection means $\sqcap$.

The literature has produced few examples of soft topologies. Beyond the trivial cases $\left(\tau_{i d}=\right.$ $\{\Phi, \tilde{X}\}$, the indiscrete soft topology, and $\tau_{d}=S S(X)$, the discrete soft topology [5], Definitions 21 and 22) and some specific examples, the only general construction we are aware of appeared in Terepeta [11].

Definition 2 ([11]). Suppose that $\Sigma$ is a crisp topology on $X$.

The soft topology on $X$ generated by $\Sigma, \tau(\Sigma)$, is the collection of all soft sets $(F, E)$ on $X$ for which every $F(e)$ lies in $\Sigma$. Formally:

$$
\tau(\Sigma)=\left\{\{(e, F(e)): e \in E\} \in S S_{E}(X) \text { such that } F(e) \in \Sigma \text { for each } e \in E\right\} .
$$

The single set soft topology on $X$ generated by $\Sigma, \hat{\tau}(\Sigma)$, is the collection of all soft sets $(F, E)$ on $X$ for which there is $T \in \Sigma$ such that every $F(e)$ coincides with T. Formally:

$$
\hat{\tau}(\Sigma)=\left\{\{(e, F(e)): e \in E\} \in S S_{E}(X) \text { such that } F(e)=F\left(e^{\prime}\right) \in \Sigma \text { for each } e, e^{\prime} \in E\right\} .
$$


The next example demonstrates the applicability of the procedure in Definition 2.

Example 1. Suppose that $\Sigma_{c}$ is the cofinite topology on $X$, also called the topology of finite complements ([3], Example 3.8 a). This is the topology that declares open those subsets of $X$ whose complements are finite, together with $\varnothing$. When $X$ is finite, it is the discrete topology. When $X$ is uncountably infinite, the topology is separable but not first-countable, therefore not second-countable [14], II.19.

The soft topology on X generated by $\Sigma_{c}$, namely, $\tau\left(\Sigma_{c}\right)$, is called the cofinite soft topology on X.

It can be characterized as follows:

$$
\begin{gathered}
\tau\left(\Sigma_{c}\right)=\left\{\{(e, F(e)): e \in E\} \in S S_{E}(X) \text { such that } X \backslash F(e) \text { is finite, for each } e \in E\right\}= \\
\left\{(F, E) \in S S_{E}(X) \text { such that }(F, E)^{c} \text { is a finite soft set }\right\}
\end{gathered}
$$

It is in this guise that the cofinite soft topology was introduced by [9], Definition 3.2. Here we just present it as an application of Definition 2.

Observe that the general construction of soft topologies given by Definition 2 can be improved by the recourse to several topologies, one for each attribute:

Corollary 1. Suppose that $\boldsymbol{\Sigma}=\left\{\Sigma_{e}\right\}_{e \in E}$ is a family of crisp topologies on $X$, indexed by a set E. Then

$$
\tau(\boldsymbol{\Sigma})=\left\{\{(e, F(e)): e \in E\} \in S S_{E}(X) \text { such that } F(e) \in \Sigma_{e} \text { for each } e \in E\right\}
$$

is a soft topology on $X$. When $\Sigma_{e}=\Sigma_{e^{\prime}}=\Sigma$ for each e, $e^{\prime} \in E, \tau(\Sigma)=\tau(\Sigma)$.

We say that $\tau(\boldsymbol{\Sigma})$ is the soft topology on $X$ generated by the family of topologies $\boldsymbol{\Sigma}$. It is the collection of all soft sets $(F, E)$ on $X$ for which every $F(e)$ lies in $\Sigma_{e}$. When all the $\Sigma_{e}$ coincide (to a common topology $\Sigma$ ) then the soft topologies on $X$ generated by the family $\Sigma$ and the topology $\Sigma$ coincide as well.

Just like bases are collections of subsets that generate topologies, soft bases are collections of soft sets that produce a soft topology by taking the set formed by all unions of their elements. The abstract concept of a soft base for a given soft topology has been known since [4].

Definition 3 ([4]). Suppose that $\tau$ is a soft topology on $X$. Then $\mathcal{B} \subseteq \tau$ is a soft base for $\tau$ when every $(F, E) \in \tau$ can be expressed as a union of soft sets from $\mathcal{B}$.

However Definition 3 cannot be used to enlarge the catalogue of actual soft topologies. Roy and Samanta [10] produced a related concept that can be eventually used to this purpose (but we believe that it has never been used to create distinguished soft topologies).

Definition 4 ([10]). Suppose that $\mathcal{B} \in \mathcal{P}\left(S S_{E}(X)\right)$ is a collection of soft sets on $X$. It is a soft open base for a soft topology on $X$, when $\mathcal{B}$ satisfies the following conditions:

(1) $\Phi \in \mathcal{B}$;

(2) the union of all soft sets in $\mathcal{B}$ is $\tilde{X}$, i.e., for each $e \in E$ and $x \in X$, there exists $\left(F_{x}, E\right) \in \mathcal{B}$ such that $x \in F_{x}(e)$; and

(3) when $\left(F_{1}, E\right),\left(F_{2}, E\right) \in \mathcal{B}$ and $x \in\left(\left(F_{1}, E\right) \sqcap\left(F_{2}, E\right)\right)(e)=F_{1}(e) \cap F_{2}(e)$, there exists $(G, E) \in \mathcal{B}$ such that $(G, E) \sqsubseteq\left(F_{1}, E\right) \sqcap\left(F_{2}, E\right)$ and $x \in G(e)$.

Roy and Samanta [10], Theorem 13 establish the soft topology generated by a soft open base $\mathcal{B}$, denoted $\tau_{\mathcal{B}}$. Their Theorem 16 proves that $\mathcal{B} \subseteq \tau_{\mathcal{B}}$ is a soft base for $\tau_{\mathcal{B}}$ in the sense of Definition 3 . Thus, the members of $\tau_{\mathcal{B}}$ are exactly the soft sets that can be written as a union of soft sets from $\mathcal{B}$. 
In other words, a soft set $(F, E)$ belongs to $\tau_{\mathcal{B}}$ if and only if there is a family $\left\{\left(F_{i}, E\right)\right\}_{i \in I}$ of members of $\mathcal{B}$ such that $(F, E)=\sqcup_{i \in I}\left(F_{i}, E\right)$. And conversely, their Theorem 17 proves that any soft base for a soft topology $\tau$ on $X$ (in the sense of Definition 3) must be a soft open base for a soft topology (in the sense of Definition 4).

Quite naturally, we say that a soft topology is soft second-countable (S2C) when there is a soft base for it with countably many elements [8], Definition 4.32. This definition is a faithful replication of the pattern of a second-countable topological space. In contrast, the notion of soft separability is controversial. The next section discusses this issue in depth.

\section{Soft Separability: New Proposal}

In the case of crisp topologies, separability amounts to the existence of a countable dense subset. Remember that when $\Sigma$ is a crisp topology on $X, U \subseteq X$ is dense in $X$ if its topological closure is $X$, i.e., if $C l(U)=X$.

Attempts have been made to define soft separability of a soft topology on $X$. The only reliable source for a definition was given by Das and Samanta [8] in the context of soft metric spaces. It mimics the standard definition in crisp topologies. However this comes at the cost of being rather difficult to handle. It may also be possible to put forward alternative definitions with a different principle. Whereas [8] builds on countable soft sets whose soft closure is the absolute soft set $\bar{X}$, one might instead rely on the existence of countable collections of soft points with a similar behavior.

At any rate, a nice concept of soft separability is requested to satisfy three desirable characteristics:

(1) It should, to some extent, replicate the spirit of separability in crisp topologies.

(2) It must be implied by S2C, which is universally accepted.

(3) It should be easy to understand and amenable to technical operations.

In our view Das and Samanta's soft separability meets the first two characteristics. It is a matter of opinion whether the third one is met, since it is highly subjective as a foundation for a concept of soft separability. Nevertheless, separability of crisp topologies is indeed simple to explain and we would expect a similarly undemanding notion in the soft context.

All things considered, we proceed to study soft separability with two principles in mind. First, the distinction between soft separabilities based on countable soft sets versus countable collections of 'soft points'. We dedicate separate subsections to each position. Secondly, a simpler approach to the concept that understands density (thus separability) as follows:

Lemma 1. Suppose that $\Sigma$ is a crisp topology on $X$, and $U \subseteq X$. Then $U$ is dense in $X$ (i.e., $C l(U)=X)$ if and only if each non-empty $V \in \Sigma$ intersects $U$ (i.e., $U \cap V \neq \varnothing$ for each $V \in \Sigma \backslash\{\varnothing\}$ ).

Therefore $\Sigma$ is a separable crisp topology on $X$ if and only if there is a countable $U \subseteq X$ such that $U \cap V \neq \varnothing$ for each $V \in \Sigma, V \neq \varnothing$.

It is this alternative (and equivalent) definition of separability that we intend to extend to the realm of soft topologies. To avoid further confusion with existing literature, our two adapted versions of topological separability will avoid this term. In this we follow Munkres [2], Chapter 4, §30, who claims that the name separability is "an unfortunate choice of terminology" and "a good example of how a word can be overused". Indeed, separation of a space and separation axioms in topology are based on very different ideas. And the same goes for soft topology. In addition, we will give persuasive arguments to recommend these ideas as convenient extensions of the idea of separability to soft topological spaces.

For further use we define a special type of soft sets. They are vaguely related to the idea of soft points in [8].

Definition 5. For any $U \subseteq X$ and $e \in E$, we define $\left(F_{U}^{e}, E\right) \in S S_{E}(X)$ such that $F_{U}^{e}(e)=U$ and $F_{U}^{e}\left(e^{\prime}\right)=\varnothing$ when $e^{\prime} \neq e$. 


\subsection{Soft Separability as Countable Soft-Set Density}

In view of Lemma 1, it is natural to extend the spirit of topological separability as follows:

Definition 6. Suppose that $\tau$ is a soft topology on $X$. We say that

$(F, E) \in S S_{E}(X)$ is $\tau$-soft dense in $X$ if $(F, E) \sqcap(G, E) \neq \Phi$, for every $(G, E) \in \tau,(G, E) \neq \Phi$; and $\tau$ is countably soft-set-dense (henceforth $C S^{2} D$ ) if there is a countable soft set that is $\tau$-soft dense in $X$.

Thus, $\mathrm{CS}^{2} \mathrm{D}$ is similar to Das and Samanta's soft separability in that it postulates the existence of a countable soft set with suitable characteristics. The difference is that our concept bypasses the appeal to additional definitions, like soft closures or soft limit points, in order to replicate the behavior of a countable dense set in crisp topologies.

To avail $C S^{2} \mathrm{D}$ as a sensible separability-type concept, we do two things. We confirm that it is implied by S2C in the next section (cf., Proposition 1). In addition, Section 3.3 establishes a strong link of $C S^{2} \mathrm{D}$ with separability in the context of standard topologies.

\subsection{Soft Separability as Soft-Points Countable Density}

An alternative way of extending topological separability-defined by Lemma 1—to soft topologies uses countable collections of 'soft points' instead of one countable soft set.

We propose to capture the spirit of topological separability in the following additional form:

Definition 7. Suppose that $\tau$ is a soft topology on $X$. We say that

$\mathcal{F} \in \mathcal{P}\left(S S_{E}(X)\right)$ is soft $\tau$-dense in $X$ if for every $(G, E) \in \tau,(G, E) \neq \Phi$, it must be the case that $(F, E) \sqcap(G, E) \neq \Phi$ for some $(F, E) \in \mathcal{F}$; and

$\tau$ is soft-points countably-dense (henceforth SPCD) if there is a countable collection $\mathcal{F}$ of soft points that is soft $\tau$-dense in $X$.

Observe that SPCD implies $C S^{2} \mathrm{D}$ :

Lemma 2. Suppose that $\tau$ is a SPCD soft topology on $X$. Then $\tau$ is $C S^{2} D$.

Proof. There exists a countable family of soft points, $\mathcal{F}=\left\{\left(\left\{x_{i}\right\}, E\right) \mid i \in I\right\}$, with $I$ countable and the property that for every $(G, E) \in \tau,(G, E) \neq \Phi$, there is $i \in I$ such that $\left(\left\{x_{i}\right\}, E\right) \sqcap(G, E) \neq \Phi$.

Consider $(F, E)$ such that $F(e)=\left\{x_{i} \mid i \in I\right\}$, a countable subset of $X$, for each $e \in E$. We have thus defined $(F, E) \in S S_{E}(X)$, a countable soft set. It is obvious that $(F, E)$ is $\tau$-soft dense.

More importantly, SPCD (thus $\mathrm{CS}^{2} \mathrm{D}$ ) is implied by S2C:

Proposition 1. Let $\tau$ be a S2C soft topology $\tau$ on X. Then $\tau$ is SPCD, thus $C S^{2} D$.

Proof. There exists a countable soft base $\mathcal{B}=\left\{\left(F_{i}, E\right) \mid i \in I\right\}$ for $\tau$. Therefore $I$ is countable and $\left(F_{i}, E\right) \in S S_{E}(X)$ for each $i \in I$.

For each $i \in I$, if $\left(F_{i}, E\right) \neq \Phi=\left(F_{i_{0}}, E\right)$ there is $e \in E$ such that $F_{i}(e) \neq \varnothing$, thus we can select $x_{i} \in F_{i}(e)$. We let $I_{0}=I \backslash\left\{i_{0}\right\}$.

Consider the countable family of soft points $\mathcal{F}=\left\{\left(\left\{x_{i}\right\}, E\right) \mid i \in I_{0}\right\}$ thus defined. We claim that it is soft $\tau$-dense in $X$, which proves that $\tau$ is SPCD.

Fix $(G, E) \in \tau,(G, E) \neq \Phi$. Because $\mathcal{B}$ is a soft base for $\tau,(G, E)=\sqcup_{i \in J}\left(F_{i}, E\right)$ for some $J \subseteq I$. Now we select $j \in J$ for which there exists $e \in E$ with $F_{j}(e) \neq \varnothing$. By construction, $\left(\left\{x_{j}\right\}, E\right) \in \mathcal{F}$. As $x_{j} \in F_{j}(e) \subseteq G(e)$, we can guarantee $\left(\left\{x_{j}\right\}, E\right) \sqcap(G, E) \neq \Phi$.

At the end of Section 3.3 we prove that S2C is not implied by SPCD. 


\subsection{Relationships with Crisp Separability}

The next two results show both that Corollary 1 is useful, and that our new notions in this section, are conveniently linked to the standard notion of separability in topology:

Proposition 2. Suppose that $\boldsymbol{\Sigma}=\left\{\Sigma_{e}\right\}_{e \in E}$ is a family of topologies on $X$, such that $\Sigma_{e^{\prime}}$ is separable for some $e^{\prime} \in E$. Then the soft topology $\tau(\boldsymbol{\Sigma})$ is SPCD, thus $C S^{2} D$.

In particular, if $\Sigma$ is separable topology on $X$ then the soft topology $\tau(\Sigma)$ on $X$ is $S P C D$ (and $C S^{2} D$ ).

Proof. Let us fix $\left\{x_{j} \mid j \in I\right\}$, a countable dense subset of $\Sigma_{e^{\prime}}$. We prove that $\left\{\left(\left\{x_{j}\right\}, E\right) \mid j \in I\right\}$ is soft $\tau(\Sigma)$-dense in $X$. To see that this countable collection of soft points satisfies the requirements of Definition 7 , let us fix an arbitrary $(F, E) \in \tau(\boldsymbol{\Sigma})$. Since $F\left(e^{\prime}\right) \in \Sigma_{e^{\prime}}$, there must exist $i \in I$ such that $x_{i} \in F\left(e^{\prime}\right)$, which implies $\left(\left\{x_{i}\right\}, E\right) \sqcap(F, E) \neq \Phi$. Therefore $\Sigma$ is SPCD.

The consequence on $\tau(\Sigma)$ when $\Sigma$ is separable is now obvious.

It is also possible to prove a converse of Proposition 2:

Proposition 3. Suppose that $\boldsymbol{\Sigma}=\left\{\Sigma_{e}\right\}_{e \in E}$ is a family of topologies on $X$, such that $\tau(\boldsymbol{\Sigma})$ is $C S^{2} D$. Then $\Sigma_{e}$ is separable for each $e \in E$. In particular, if $\Sigma$ is a topology on $X$ such that the soft topology $\tau(\Sigma)$ on $X$ is $C S^{2} D$ (or SPCD) then $\Sigma$ is separable.

Proof. Let us fix $(F, E)$, a countable $\tau(\boldsymbol{\Sigma})$-soft dense set in $X$. We claim that for each $e \in E, F(e)$ is dense in $X$ with the $\Sigma_{e}$ topology. Note that by definition, it is a countable subset of $X$.

We appeal to Lemma 1. Let us fix $U \in \Sigma_{e}$. We define the soft set $\left(F_{U}^{e}, E\right) \in \tau(\boldsymbol{\Sigma})$ (cf., Definition 5). Because $(F, E)$ is $\tau(\boldsymbol{\Sigma})$-soft dense, it must be the case that $(F, E) \sqcap\left(F_{U}^{e}, E\right) \neq \Phi$. This necessarily entails $F(e) \cap U \neq \varnothing$.

The consequence on $\tau(\Sigma)$ is now obvious.

Finally, Example 1 help us to check that S2C is indeed different from SPCD. Suppose that $E=\{e\}$ and that $X$ is uncountable. Then $\tau\left(\Sigma_{c}\right)$, the cofinite soft topology on $X$, is SPCD because $\Sigma_{c}$, the crisp cofinite topology on $X$, is separable and we can invoke Proposition 2 . The fact that $\Sigma_{c}$ is not second-countable easily proves that $\tau\left(\Sigma_{c}\right)$ cannot be S2C. Nevertheless this fact can also be established by an appeal to Corollary 2 below.

\section{A Characterization of Soft Second-Countability for Soft Topologies}

Second-countable crisp topologies are those that have countable bases. It is straightforward to extend the concept to soft topologies: remember that a soft topology with a countable soft base is a soft second-countable or S2C soft topology. This concept is superfluous when both $X$ and $E$ are finite because in this case, all soft topologies are finite (i.e., formed by a finite number of soft sets) thus countable; and countable soft topologies are obviously S2C. In this section we avail ourselves of the ideas in Section 5.1 in order to produce the first non-trivial soft second-countable soft topologies in the literature.

Theorem 1. Let $\Sigma$ be a second-countable topology on $X$. Then when $E$ is finite, $\tau(\Sigma)$ is $S 2 C$.

Proof. Let $\beta$ be a countable base for $\Sigma$. Theorem 3 ensures that $\tau(\Sigma)=\tau_{\mathcal{B}(\beta)}$ thus $\mathcal{B}(\beta)$ is a soft open base for $\tau(\Sigma)$ by [10], Theorem 16. We just need to observe that $\mathcal{B}(\beta)$ is countable when $E$ is finite.

In fact, a direct arguments proves an improvement of Theorem 1:

Theorem 2. Let $\Sigma=\left\{\Sigma_{e}\right\}_{e \in E}$ be a family of topologies on $X$, such that $\Sigma_{e}$ is second-countable for each $e \in E$. Then when $E$ is countable, $\tau(\boldsymbol{\Sigma})$ is $S 2 C$. 
Proof. Let $\left\{\beta^{e}\right\}$ be a countable base for $\Sigma_{e}$, for each $e \in E$. We claim that

$$
\mathcal{B}=\left\{\left(F_{U}^{e}, E\right) \text { such that } U \in \beta^{e} \text { for each } e \in E\right\}=\cup_{e \in E}\left\{\left(F_{U}^{e}, E\right) \text { such that } U \in \beta^{e}\right\}
$$

is a countable soft base for $\tau(\Sigma)$. It is a countable family because $E$ is countable, each $\left\{\beta^{e}\right\}$ is countable, and the countable union of countable sets is countable. Its members belong to $\tau(\Sigma)$ by definition of this soft topology. To prove that $\mathcal{B}$ is a soft base of $\tau(\boldsymbol{\Sigma})$, let us fix $(F, E) \in \tau(\boldsymbol{\Sigma})$, i.e., $F(e) \in \Sigma_{e}$ for each $e \in E$.

Then there exist $\left\{U_{i}\right\}_{i \in I(e)}$, respective subfamilies of open sets in $\beta^{e}$ with the property that $F(e)=\cup_{i \in I(e)} U_{i}$ for each $e \in E$. Note that for each $U \in\left\{U_{i}\right\}_{i \in I(e)}$ and $e \in E$, the soft set $\left(F_{U}^{e}, E\right)$ belongs to $\mathcal{B}$ by definition.

The reader can easily check that $\mathcal{F}=\left\{\left(F_{U}^{e}, E\right)\right.$ such that $U \in\left\{U_{i}\right\}_{i \in I(e)}$ for each $\left.e \in E\right\} \subseteq \mathcal{B}$ satisfies $(F, E)=\sqcup\left\{\left(F_{U}^{e}, E\right) \mid\left(F_{U}^{e}, E\right) \in \mathcal{F}\right\}$.

We can also prove a converse to this result. Its proof follows that of Proposition 3 closely.

Proposition 4. Suppose that $\Sigma=\left\{\Sigma_{e}\right\}_{e \in E}$ is a family of topologies on $X$, such that $\tau(\Sigma)$ is S2C. Then $\Sigma_{e}$ is second-countable for each $e \in E$. In particular, if $\Sigma$ is a topology on $X$ such that $\tau(\Sigma)$ is $S 2 C$ then $\Sigma$ is second-countable.

Proof. Let us select $\left\{\left(F_{i}, E\right)\right\}_{i \in I}$, a countable soft base of $\tau(\Sigma)$ in $X$. We claim that for each $e \in E$, the countable collection $\left\{F_{i}(e)\right\}_{i \in I}$ is a base of the $\Sigma_{e}$ topology. Note that $F_{i}(e) \in \Sigma_{e}$ for each $i \in I$ and $e \in E$, by definition of $\tau(\Sigma)$.

Let us fix $U \in \Sigma_{e}$. We define the soft set $\left(F_{U}^{e}, E\right)$ as in Definition 5, then $\left(F_{U}^{e}, E\right) \in \tau(\Sigma)$. Because $\left\{\left(F_{i}, E\right)\right\}_{i \in I}$ is a soft base of $\tau(\Sigma),\left(F_{U}^{e}, E\right)=\sqcup_{j \in J}\left(F_{j}, E\right)$ for some subfamily $J \in I$. This necessarily entails $F_{U}^{e}(e)=U=\cup_{j \in J} F_{j}(e)$. We conclude that $\left\{F_{i}(e)\right\}_{i \in I}$ is a base of $\Sigma_{e}$.

The particular consequence for $\tau(\Sigma)$ is now obvious.

If we combine Theorem 2 and Proposition 4 we readily deduce a very appealing property of the soft topology defined in Corollary 1:

Corollary 2. Suppose that $\Sigma=\left\{\Sigma_{e}\right\}_{e \in E}$ is a family of topologies on $X$, and $E$ is a countable set of attributes. Then $\tau(\boldsymbol{\Sigma})$ is a S2C soft topology on $X$ if and only if $\Sigma_{e}$ is second-countable for each $e \in E$.

\section{A New Constructive Method of Soft Topologies}

This section introduces a novel procedure for the construction of soft topologies. It builds on the concept of a base for a crisp topology. We investigate some fundamental properties of this mechanism.

\subsection{Soft Topologies Generated by Bases for Crisp Topologies}

Our first result in this section shows how any base for a crisp topology on $X$ generates a soft topology on $X$, for any fixed set of attributes $E$.

Proposition 5. Let $\beta$ be a base for a topology $\Sigma$ on $X$. Then the collection of soft sets on $X$

$$
\mathcal{B}(\beta)=\left\{\{(e, F(e)): e \in E\} \in S S_{E}(X) \text { such that } F(e) \in \beta \cup\{\varnothing\} \text { for each } e \in E\right\}
$$

is a soft open base for a soft topology on X.

Proof. We prove that $\mathcal{B}(\beta)$ satisfies the three requirements of Definition 4 . Actually, $\mathcal{B}(\beta)$ agrees with (1) and (3) in that Definition, and also with a property implying (2).

Note that $\Phi=\{(e, \Phi(e)): e \in E\}$ satisfies $\Phi(e)=\varnothing$, therefore it is clearly a member of $\mathcal{B}(\beta)$ by construction. This ensures (1). 
We claim that for each $x \in X$, there exists $\left(F_{x}, E\right) \in \mathcal{B}(\beta)$ such that $(\{x\}, E) \sqsubseteq\left(F_{x}, E\right)$. Observe that this property of $\mathcal{B}(\beta)$ is stronger than (2). Let us fix $x \in X$. There exists $x \in B_{x} \in \beta$ therefore if we define $\left(F_{x}, E\right)$ by $F_{x}(e)=B_{x}$ for each $e \in E$, then $(\{x\}, E) \sqsubseteq\left(F_{x}, E\right) \in \mathcal{B}(\beta)$ by definition.

In order to prove (3), suppose that $\left(F_{1}, E\right),\left(F_{2}, E\right) \in \mathcal{B}(\beta)$ and $x \in\left(\left(F_{1}, E\right) \sqcap\left(F_{2}, E\right)\right)(e)=$ $F_{1}(e) \cap F_{2}(e)$. For each $e^{\prime} \in, e^{\prime} \neq e$, we define $G\left(e^{\prime}\right)=\varnothing$. Note that $F_{1}(e), F_{2}(e) \in \beta \cup\{\varnothing\}$ by construction, $\beta$ is a base for a topology on $X$ by assumption, and $x \in F_{1}(e) \cap F_{2}(e) \neq \varnothing$. Therefore there exists $G(e) \in \beta$ such that $x \in G(e) \subseteq F_{1}(e) \cap F_{2}(e)$. We have thus defined $(G, E) \in \mathcal{B}(\beta)$ with the properties $(G, E) \sqsubseteq\left(F_{1}, E\right) \sqcap\left(F_{2}, E\right)$ and $x \in G(e)$.

Actually, a straightforward modification of the proof of Proposition 5 assures that we can produce a more general construction than the procedure that it proposes.

Corollary 3. Suppose that $\bar{\beta}=\left\{\beta_{e}\right\}_{e \in E}$ is a family of bases for respective topologies on $X$, indexed by a set E. Then $\mathcal{B}(\bar{\beta})=\left\{\{(e, F(e)): e \in E\} \in S S_{E}(X)\right.$ such that $F(e) \in \beta_{e} \cup\{\varnothing\}$ for each $\left.e \in E\right\}$ is a soft open base for a soft topology on $X$.

To demonstrate the implementability of the above Corollary, we consider the following toy example.

Example 2. Let $X=\{x, y, z\}$. Two bases for respective topologies on $X$ are $\beta_{e_{1}}=\{\varnothing, X,\{y\}\}$ and $\beta_{e_{2}}=$ $\{\varnothing, X,\{y, z\}\}$. Define $E=\left\{e_{1}, e_{2}\right\}$. Then Corollary 3 assures that a soft open base for a soft topology on $X$ is defined as the collection of the nine soft sets represented by Table 1. The first one is $\Phi_{E}$ whereas the last one is $\tilde{X}$.

Table 1. Tabular representations of the nine members of the soft open base in Example 2.

\begin{tabular}{ccccccccccccccccccc}
\hline$X$ & $e_{1}$ & $e_{2}$ & $e_{1}$ & $e_{2}$ & $e_{1}$ & $e_{2}$ & $e_{1}$ & $e_{2}$ & $e_{1}$ & $e_{2}$ & $e_{1}$ & $e_{2}$ & $e_{1}$ & $e_{2}$ & $e_{1}$ & $e_{2}$ & $e_{1}$ & $e_{2}$ \\
\hline$x$ & 0 & 0 & 0 & 0 & 0 & 1 & 0 & 0 & 0 & 0 & 0 & 1 & 1 & 0 & 1 & 0 & 1 & 1 \\
$y$ & 0 & 0 & 0 & 1 & 0 & 1 & 1 & 0 & 1 & 1 & 1 & 1 & 1 & 0 & 1 & 1 & 1 & 1 \\
$z$ & 0 & 0 & 0 & 1 & 0 & 1 & 0 & 0 & 0 & 1 & 0 & 1 & 1 & 0 & 1 & 1 & 1 & 1 \\
\hline
\end{tabular}

We are ready to formulate a problem whose solution constitutes the main result of this section. Suppose that $\beta$ is a base for a (crisp) topology $\Sigma$ on $X$. From these items we can produce two apparently different soft topologies on $X$, namely, $\tau(\Sigma)$ and $\tau_{\mathcal{B}(\beta)}$. Definition 2 gives $\tau(\Sigma)$. Roy and Samanta [10], Theorems 13,16 give $\tau_{\mathcal{B}(\beta)}$ by virtue of Proposition 5. Our next theorem proves that both soft topologies on $X$ coincide.

Theorem 3. Let $\beta$ be a base for a (crisp) topology $\Sigma$ on $X$. Then $\tau(\Sigma)=\tau_{\mathcal{B}(\beta)}$.

Proof. We prove by double inclusion the set identity $\tau(\Sigma)=\tau_{\mathcal{B}(\beta)}$.

Recall that Roy and Samanta [10], Theorem 16 establish that $\mathcal{B}(\beta)$ is a soft base for $\tau_{\mathcal{B}(\beta)}$ in the sense of Definition 3. Thus, the members of $\tau_{\mathcal{B}(\beta)}$ are exactly the soft sets that can be written as a union of soft sets from $\mathcal{B}(\beta)$. In other words, a soft set $(F, E)$ belongs to $\tau_{\mathcal{B}(\beta)}$ if and only if there is a family $\left\{\left(F_{i}, E\right)\right\}_{i \in I}$ of members of $\mathcal{B}(\beta)$ such that $(F, E)=\sqcup_{i \in I}\left(F_{i}, E\right)$.

In order to prove $\tau(\Sigma) \subseteq \tau_{\mathcal{B}(\beta)}$, suppose $(F, E) \in \tau(\Sigma)$. If we write $(F, E)=\{(e, F(e)): e \in E\}$ then the definition of $\tau(\Sigma)$ implies $F(e) \in \Sigma$ for each $e \in E$. Because $\beta$ is a base for $\Sigma$, with each $e \in E$ we can associate a family $\left\{G_{i}(e)\right\}_{i \in I(e)}$ of members of $\beta$ such that $F(e)=\cup_{i \in I(e)} G_{i}(e)$. Let us define a family of soft sets on $X$ that belong to $\mathcal{B}(\beta)$, as follows:

$$
\left\{\left(F_{i}, E\right)\right\}_{i \in I}=\left\{\left(F^{\prime}, E\right)=\left\{\left(e, F^{\prime}(e)\right): e \in E\right\} \in S S_{E}(X) \text { such that } F^{\prime}(e)=G_{i}(e) \text {, some } i \in I(e)\right\} \text {. }
$$

Because each $\left(F^{\prime}, E\right)$ in this family belongs to $\mathcal{B}(\beta)$ by construction, and it is easy to check for the equality $(F, E)=\sqcup_{i \in I}\left(F_{i}, E\right)$, we can conclude $(F, E) \in \tau_{\mathcal{B}(\beta)}$. 
Conversely, let us fix $(F, E) \in \tau_{\mathcal{B}(\beta)}$. There is $\left\{\left(F_{i}, E\right)\right\}_{i \in I} \subseteq \mathcal{B}(\beta)$ such that $(F, E)=\sqcup_{i \in I}\left(F_{i}, E\right)$. By definition of $\mathcal{B}(\beta), F_{i}(e) \in \beta \cup\{\varnothing\}$ for each $e \in E$ and $i \in I$. By definition of $\sqcup,(F, E)$ must satisfy $F(e)=\cup_{i \in I} F_{i}(e) \in \Sigma$ for each $e \in E$. This in turn assures $(F, E) \in \tau(\Sigma)$ by definition of $\tau(\Sigma)$.

The diagram in Figure 2 summarizes the elements that produce Theorem 3.

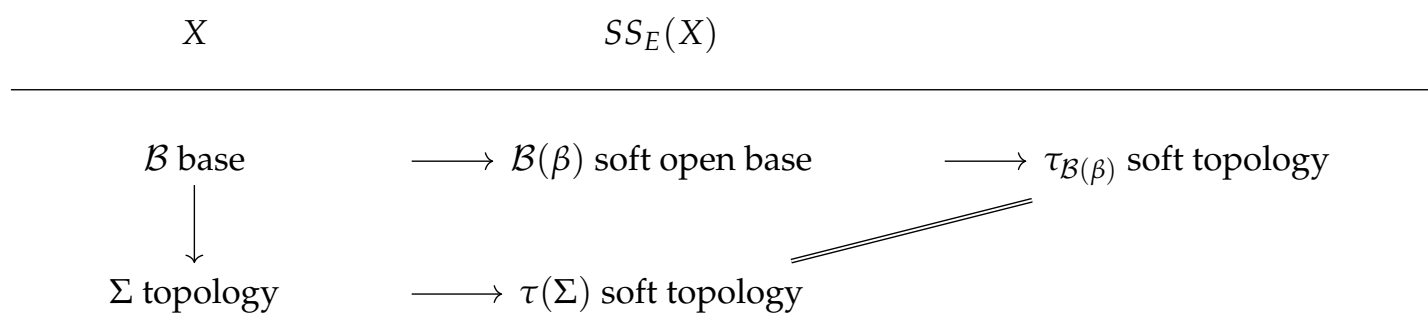

Figure 2. The commutativity diagram involving the two soft topologies in Theorem 3.

\subsection{New Non-Trivial Soft Second-Countable Soft Topologies}

Let us now show that the combination of Theorems 1 and 3 permits to define non-trivial examples of S2C soft topologies. The next example focuses on countable sets $X$.

Example 3. Let $X=\mathbb{N}$. Bases for respective crisp topologies on $X$ are

$\beta_{1}=\{\{n, n+1, \ldots\} \mid n \in \mathbb{N}\}$ [3], Problem 5.B, Chapter 2, which is clearly countable (thus the topology $\Sigma_{1}$ that it generates is second-countable); and

$\beta_{F}=\{A \subset \mathbb{N} \mid$ either $1 \notin A$ or $\mathbb{N} \backslash A$ is finite $\}$. This is a base for a countable Fort topology on $X, \Sigma_{F}$, which is second-countable [14], II.24.

Let $Y=\{2,3,4 \ldots\} \subseteq \mathbb{N}$. Consider for each $n \geqslant 2$, the set of the form $B_{n}=\{x \in \mathbb{N} \mid x$ divides $n\}$. The family $\beta_{D}=\left\{U_{n}\right\}_{n \geqslant 2}$ is a base for the divisor topology on $Y, \Sigma_{D}$, which is second-countable [14], II.57 because all elements in the base are finite thus the base itself is countable.

Thus, for any countable set of attributes $E, S 2 C$ soft topologies on $\mathbb{N}$ are $\tau\left(\Sigma_{1}\right)=\tau_{\mathcal{B}\left(\beta_{1}\right)}$ and $\tau\left(\Sigma_{F}\right)=$ $\tau_{\mathcal{B}\left(\beta_{F}\right)}$. And a S2C soft topology on $Y$ is $\tau\left(\Sigma_{D}\right)=\tau_{\mathcal{B}\left(\beta_{D}\right)}$. The argument in the proof of Theorem 2 provides formal expressions of respective countable soft bases of these soft topologies.

Actually, we can assure that when $E$ is finite, $\mathcal{B}\left(\beta_{1}\right)$ is a countable soft open base of $\tau\left(\Sigma_{1}\right)$, and that $\mathcal{B}\left(\beta_{D}\right)$ is a countable soft open base of $\tau\left(\Sigma_{D}\right)$. The argument in the proof of Theorem 1 proves this claim.

Table 2 displays a summary of the tabular representations of the elements in $\mathcal{B}\left(\beta_{1}\right)$, the countable soft open base of the soft topology $\tau\left(\Sigma_{1}\right)$ on $X$, when $E=\left\{e_{1}, e_{2}\right\}$.

One can also use the procedure above to produce S2C soft topologies on uncountable sets of alternatives. For example, the Euclidean topology $\Sigma_{e}$ on $X=\mathbb{R}$ is second-countable so with any countable set of attributes $E$ we can assure that $\tau\left(\Sigma_{e}\right)$ is a S2C soft topology on $\mathbb{R}$. In fact with each countable base for $\Sigma_{e}$, e.g., the collection $\beta_{e}$ of open intervals with rational extremes, we can associate a countable soft open base for $\tau\left(\Sigma_{e}\right)$ (see the argument in the proof of Theorem 2). When $E$ is finite, $\mathcal{B}\left(\beta_{e}\right)$ for $\tau\left(\Sigma_{e}\right)$ is another countable soft open base for $\tau\left(\Sigma_{e}\right)$. 
Table 2. Tabular representations of the countably many members of the soft open base $\mathcal{B}\left(\beta_{1}\right)$ for a soft topology on $X=\mathbb{N}$ in Example 3: A summary.

\begin{tabular}{ccccccccccccccccc}
\hline $\boldsymbol{X}$ & $e_{1}$ & $e_{2}$ & $e_{1}$ & $e_{2}$ & $e_{1}$ & $e_{2}$ & $e_{1}$ & $e_{2}$ & $\ldots$ & $e_{1}$ & $e_{2}$ & $e_{1}$ & $e_{2}$ & $e_{1}$ & $e_{2}$ & \\
\hline 1 & 0 & 0 & 0 & 1 & 0 & 0 & 0 & 0 & $\ldots$ & 1 & 1 & 1 & 0 & 1 & 0 & $\ldots$ \\
2 & 0 & 0 & 0 & 1 & 0 & 1 & 0 & 0 & $\ldots$ & 1 & 1 & 1 & 1 & 1 & 0 & $\ldots$ \\
3 & 0 & 0 & 0 & 1 & 0 & 1 & 0 & 1 & $\ldots$ & 1 & 1 & 1 & 1 & 1 & 1 & $\ldots$ \\
$\vdots$ & $\vdots$ & $\vdots$ & $\vdots$ & $\vdots$ & $\vdots$ & $\vdots$ & $\vdots$ & $\vdots$ & $\vdots$ & $\vdots$ & $\vdots$ & $\vdots$ & $\vdots$ & $\vdots$ & & \\
$n$ & 0 & 0 & 0 & 1 & 0 & 1 & 0 & 1 & $\ldots$ & 1 & 1 & 1 & 1 & 1 & 1 & $\ldots$ \\
$\vdots$ & $\vdots$ & $\vdots$ & $\vdots$ & $\vdots$ & $\vdots$ & $\vdots$ & $\vdots$ & $\vdots$ & $\vdots$ & $\vdots$ & $\vdots$ & $\vdots$ & $\vdots$ & $\vdots$ & & \\
\hline$X$ & $e_{1}$ & $e_{2}$ & $e_{1}$ & $e_{2}$ & $e_{1}$ & $e_{2}$ & $e_{1}$ & $e_{2}$ & $\ldots$ & & & & & & \\
\hline 1 & 0 & 0 & 0 & 1 & 0 & 0 & 0 & 0 & $\ldots$ & & & & & & \\
2 & 1 & 0 & 1 & 1 & 1 & 1 & 1 & 0 & $\ldots$ & & & & & & \\
3 & 1 & 0 & 1 & 1 & 1 & 1 & 1 & 1 & $\ldots$ & & & & & & & \\
$\vdots$ & $\vdots$ & $\vdots$ & $\vdots$ & $\vdots$ & $\vdots$ & $\vdots$ & $\vdots$ & $\vdots$ & $\ldots$ & & & & & & & \\
$n$ & 1 & 0 & 1 & 1 & 1 & 1 & 1 & 1 & $\ldots$ & & & & & & & \\
$\vdots$ & $\vdots$ & $\vdots$ & $\vdots$ & $\vdots$ & $\vdots$ & $\vdots$ & $\vdots$ & $\vdots$ & $\ldots$ & & & & & & & \\
\hline
\end{tabular}

\section{Conclusions}

This paper shows that the research about soft topological spaces is an active field, where fundamental contributions can still be made. Consequently, it presents strictly theoretical considerations on soft topologies.

The development of topology has been nurtured by the continuous supply of examples and classes of topological spaces. It is, therefore, important to enlarge the list of soft topological spaces, and of their properties and relations. We have contributed to this constituent of soft topology with some new classes of soft topological spaces. We have produced non-trivial examples of soft second-countable soft topologies. In fact, countable soft open bases of these soft topologies have been identified.

Relatedly, two new concepts of 'soft separability' have been proposed. They are implied by (but different from) soft second-countability, and they are simple to understand and operate with. Further technical arguments show that they can play a part in the field of soft topology.

We have reexamined a general procedure for generating soft topologies from crisp topologies, that has been known since [11]. It has been extended in two directions. First, by allowing that different attributes are associated with different crisp topologies. Secondly, by the recourse to the simpler notion of a base, and again we use one base for each attribute. The first procedure preserves separability under the sole assumption that one of the attributes is associated with a separable crisp topology. Its behavior with respect to second-countability is still better, and an 'if-and-only-if' result has been achieved. The second procedure gives rise to a commutativity diagram involving the original procedure: if we generate a soft topology from a base for a topology, and we generate a soft topology from the topology that the base produces, both soft topologies are the same. Thus, it may be argued that the second procedure allows to operationalize the well-established procedure for the generation of soft topologies in [11].

Practical applications are beyond the scope of this article. The new strategies for the production of soft topologies may be applied in the future in the analysis of other properties, like the soft separation axioms (soft normality, soft regularity, $T_{0}, T_{1}, T_{2}, T_{3}, T_{4}, \ldots$ ) in $[5,11]$.

Funding: This work has received no external funding.

Acknowledgments: The author is highly grateful to two anonymous reviewers for their valuable comments and helpful suggestions.

Conflicts of Interest: The author declares no conflict of interest. 


\section{References}

1. Molodtsov, D. Soft set theory-First results. Comput. Math. Appl. 1999, 37, 19-31. [CrossRef]

2. Munkres, J. Topology; Featured Titles for Topology; Prentice Hall, Incorporated: Upper Saddle River, NJ, USA, 2000.

3. Willard, S. General Topology; Addison-Wesley Series in Mathematics; Dover Publications: Mineola, NY, USA, 2004.

4. Çă̆man, N.; Karataş, S.; Enginoglu, S. Soft topology. Comput. Math. Appl. 2011, 62, 351-358. [CrossRef]

5. Shabir, M.; Naz, M. On soft topological spaces. Comput. Math. Appl. 2011, 61, 1786-1799. [CrossRef]

6. Abbas, M.; Murtaza, G.; Romaguera, S. On the fixed point theory of soft metric spaces. Fixed Point Theory Appl. 2016, 2016, 17. [CrossRef]

7. Aygünoğlu, A.; Aygün, H. Some notes on soft topological spaces. Neural Comput. Appl. 2012, 21, 113-119. [CrossRef]

8. Das, S.; Samanta, S. Soft metric. Ann. Fuzzy Math. Inf. 2013, 6, 77-94.

9. Nazmul, S.; Samanta, S. Some properties of soft topologies and group soft topologies. Ann. Fuzzy Math. Inf. 2014, 8, 645-661.

10. Roy, S.; Samanta, T.K. A note on a soft topological space. Punjab Univ. J. Math. 2014, 46, $19-24$.

11. Terepeta, M. On separating axioms and similarity of soft topological spaces. Soft Comput. 2019, 23, $1049-1057$. [CrossRef]

12. Maji, P.; Biswas, R.; Roy, A. Soft set theory. Comput. Math. Appl. 2003, 45, 555-562. [CrossRef]

13. Çağman, N.; Enginoğlu, S. Soft set theory and uni-int decision making. Eur. J. Oper. Res. 2010, 207, 848-855. [CrossRef]

14. Steen, L.; Seebach, J. Counterexamples in Topology; Dover Books on Mathematics; Dover Publications: Mineola, NY, USA, 2013.

(C) 2020 by the author. Licensee MDPI, Basel, Switzerland. This article is an open access article distributed under the terms and conditions of the Creative Commons Attribution (CC BY) license (http://creativecommons.org/licenses/by/4.0/). 